\title{
Pyrazinamide and Pyrazinoic Acid Activity against Tubercle Bacilli in Cultured Human Macrophages and in the BACTEC System
}

\author{
Max Salfinger,* Alfred J. Crowle, \\ and L. Barth Reller*
}

\begin{abstract}
From the Webb-Waring Lung Institute, the Department of Microbiology and Immunology, and the Clinical Microbiology Laboratory, University of Colorado Health Sciences Center, Denver
\end{abstract}

\begin{abstract}
Pyrazinamide (PZA) has become an essential component of current 6-month regimens for therapy of tuberculosis. Susceptible strains of tubercle bacilli convert PZA to pyrazinoic acid (POA) through pyrazinamidase (PZase), which resistant strains and Mycobacterium bovis bacille Calmette-Guérin lack. PZA susceptibility results obtained in cultured human macrophages were compared with those in the broth BACTEC system with $7 \mathrm{H12}$ medium at pH 6.0 for strains known to be PZasepositive or -negative. Although added POA was unable to inhibit tubercle bacilli in cultured macrophages, it was able to inhibit them at very high concentrations in the BACTEC broth. Intracellularly formed POA would not be able to escape from the macrophage, and therefore would accumulate sufficiently to lower $\mathrm{pH}$ to toxic levels for tubercle bacilli. The results suggest that the cultured macrophages contribute actively or passively to the effectiveness of PZA, such as through the proposed mechanism of low $\mathrm{pH}$ generated by PZase in the phagolysosomes.
\end{abstract}

In 1982 the World Health Organization estimated that 4-5 million new highly infectious, smear-positive cases of pulmonary infection with Mycobacterium tuberculosis occur worldwide every year [1].

Although pyrazinamide (PZA) was synthesized before 1940 [2], its antituberculous activity was not recognized until the early 1950s [3]. Currently, PZA is one of the essential drugs in the modern chemotherapy of tuberculosis $[4,5]$. The inclusion of PZA in treatment regimens has made possible the shortening of therapy to 6 months [6,7]. Analyzing data from several clinical trials with different regimens, Mitchison [8] hypothesized that antituberculous drug activity is influenced by different mycobacterial populations within lesions. The populations are defined by the metabolic activity of the tubercle bacilli and the $\mathrm{pH}$ of their microenvironment.

Konno et al. [9] found a correlation between pyrazinamidase (PZase) activity and susceptibility of Mycobacterium bovis and $M$. tuberculosis to PZA. PZA-susceptible strains of $M$. tuberculosis deaminate PZA to pyrazinoic acid (POA), whereas PZA-resistant strains of $M$. tuberculosis and the naturally resistant $M$. bovis strains are not able to convert PZA to POA.

Recently, a simple radiometric method was reported for susceptibility testing of $M$. tuberculosis against PZA at $\mathrm{pH} 6.0$

\footnotetext{
Received 8 November 1989; revised 26 January 1990.

Informed consent was obtained from study subjects.

Reprints and correspondence: Dr. Max Salfinger, Department of Medical Microbiology, University of Zurich, Gloriastrasse 30, CH-8028 Zurich, Switzerland.

* Present addresses: Department of Medical Microbiology, University of Zurich (M. S.) and Departments of Pathology and Medicine, Duke University Medical Center, Durham, NC (L. B. R.).

The Journal of Infectious Diseases 1990;162:201-207

(c) 1990 by The University of Chicago. All rights reserved.

$0022-1899 / 90 / 6201-0030 \$ 01.00$
}

[10]. This BACTEC method and the in vitro system of cultured human blood macrophages [11] made possible more definitive experiments with PZA.

The aims of the present study were to ascertain whether cultured human blood macrophages could be used to study the interaction of PZA with different clinical strains of tubercle bacilli, to compare those results with the minimum inhibitory concentrations (MICs) determined in the BACTEC system, and to study the mechanism of PZA action in both systems.

\section{Materials and Methods}

Human subjects. Blood monocytes [12] from a healthy tuberculin purified protein derivative (PPD)-negative white woman and from a healthy PPD-positive white man, both 38 years old, were used.

Tubercle bacilli. Nine mycobacterial isolates were used: four clinical $M$. tuberculosis isolates from different patients (stock culture numbers TB186, TB189, TB206, and TB213), three laboratory strains (Erdman, H37, and PZA-resistant [TMC311]), and 2 different sources of $M$. bovis bacille Calmette-Guérin (BCG): Paris 1173 derivative (BCG-French) and macrophage-passaged BCG originally of the Tice strain (BCG-WWLI) [13]. All three laboratory strains and TB213 were fully susceptible to isoniazid, rifampin, ethambutol, and streptomycin. TB186 was monoresistant to rifampin and TB189 to isoniazid, and TB206 was resistant to both. The isolates were prepared in batches and stored in single-use aliquots at $-90^{\circ} \mathrm{C}$ until being used to infect the macrophages. The preparation and use of the Erdman strain was as described [11], except that the inoculum for the macrophages was reduced to $\sim 100-300$ colony-forming units (cfu) $/ \mathrm{ml}$. The others were prepared similarly.

Macrophage cultures. The human monocytes used were adherent cells from peripheral blood cultured in monolayers with $\sim 5 x$ $10^{5}$ cells in each of three spots on disposable plastic Petri dishes (35 mm diameter). Each dish contained $1.5 \mathrm{ml}$ of RPMI 1640 medium supplemented with $1 \%$ unheated $\mathrm{AB}$ serum and kept at $\mathrm{pH} 7.2$ in $7.5 \% \mathrm{CO}_{2}$. The monocytes were cultured for 7 days, and the result- 
ing macrophages were infected for $30 \mathrm{~min}$ at $37^{\circ} \mathrm{C}$ with ultrasonically dispersed, log-phase mycobacteria. After infection, the monocytes were washed twice with unsupplemented, prewarmed RPMI and then incubated for 7 more days (for $M$. bovis BCG strains, 10 days) in complete RPMI medium with or without the tested drugs as described below. The controls were done in duplicate or triplicate. In one experiment, media from 7-day-old macrophage cultures without drugs were filter-sterilized and used again ("conditioned medium").

Counts of bacilli. Counts were made using samples of Petri dish cultures taken at 0 (immediately after infection), 4, and 7 days (unless otherwise stated) as described [12]. Briefly, medium from each plate was drawn off and discarded. The plates were rinsed twice with 7H9 liquid medium (Difco, Detroit) and frozen at $-90^{\circ} \mathrm{C}$. When all plates were collected, each was thawed and lysed with sodium dodecyl sulfate solution. The lysate was neutralized with bovine serum albumin, sonicated for $10 \mathrm{~s}$, and diluted in 10-fold steps in $7 \mathrm{H} 9$ medium. Samples were plated in quintuplet on 7H10 agar.

After 2 weeks of incubation, colonies of mycobacteria on the 7H10 agar plates were counted, and from these counts the numbers of culturable mycobacteria per $1 \times 10^{5}$ macrophages was calculated [11]. The values reported were means of five values each. Standard errors of means usually were $<10 \%$ of the means; these values are not shown in the figures, because the data are plotted semilogarithmically.

We used three terms to describe the effect of the drugs: bacteriostatic, no change in number of viable bacilli over time compared with the initial inoculum; inhibitory, increased number of viable bacilli compared with the initial inoculum but fewer than the control; and bactericidal, decreased number of viable bacilli over time compared with the initial inoculum.

Drugs. Pyrazinamide (Sigma Chemical, St. Louis) and pyrazinoic acid (Aldrich Chemical, Milwaukee) were dissolved and diluted in the RPMI culture medium to the required concentrations of PZA (25-800 mg/l) and POA (12.5-200 mg/l) before sterilization by membrane filtration.

$M I C$ determinations in the BACTEC system [10]. Isolates were cultured in regular 7H12 broth (Becton Dickinson Diagnostic Instrument Systems, Towson, MD) supplemented with polyoxyethylene stearate (POES) at $36^{\circ} \mathrm{C}$. The growth index was recorded daily in the BACTEC system until it was $>300$. Then, $0.1 \mathrm{ml}$ of this cul- ture was transferred into a special $7 \mathrm{Hl} 2$ broth $(4 \mathrm{ml}, \mathrm{pH} 6.0$; Becton Dickinson) that contained 25,50 , and $100 \mathrm{mg} / \mathrm{l}$ of PZA after addition of $0.1 \mathrm{ml}$ of the appropriate stock solution of PZA with POES. This set included controls at $\mathrm{pH} 6.8$ and $\mathrm{pH} 6.0$. Isolates resistant to $100 \mathrm{mg} / \mathrm{l} \mathrm{PZA}$ were retested against $25-100$ and $200-6400 \mathrm{mg} / \mathrm{l}$. The growth in all vials was recorded daily until the growth index in the control vial $(\mathrm{pH} 6.0)$ reached $\geqslant 200$. The MIC was defined as the lowest concentration that produced $\leqslant 10 \%$ of the growth index compared with the control vial.

Killing curves. Since large amounts of PZA are not dissolved easily, $2 \mathrm{ml}$ of the special $7 \mathrm{H} 12$ broth ( $\mathrm{pH} 6.0$ ) was used for preparation of the stock solutions of PZA. The other $2 \mathrm{ml}$ of broth was inoculated with the Erdman strain and incubated. At day 2, the growth index was $\sim 60$, and $2 \mathrm{ml}$ of broth (with or without PZA) was added to all vials, resulting in a final concentration of $0,25,100,400$, or $1600 \mathrm{mg} / \mathrm{l}$ PZA. This set was used in duplicate (10 vials). Immediately after the $2 \mathrm{ml}$ of broth was added (day 0 ), an aliquot of 0.1 $\mathrm{ml}$ was taken and diluted with regular 7H9 broth in a microtiter plate. From appropriate dilutions, $20 \mu \mathrm{l}$ of the broth was spotted in quintuplicate onto a $7 \mathrm{H10}$ agar plate, and after 2 weeks of incubation the colonies were counted and the colony-forming units per milliliter calculated. To minimize the loss of broth during the experiment, an aliquot was taken only every other day until day 8 . All vials were measured for ${ }^{14} \mathrm{CO}_{2}$ production in the BACTEC system.

PZase activity. PZase activity was determined by the method described by Wayne [14] with heavily grown cultures on LöwensteinJensen slants. Isolates without PZase activity at day 7 were tested twice before being considered negative.

Determination of $\mathrm{pH}$ of broths. The $\mathrm{pH}$ was determined with a digital pH meter (Corning Model 130 with Semi-Micro Combination Probe, No. 476541).

\section{Results}

Effect of PZA against different $\mathrm{M}$. tuberculosis isolates in macrophages. The virulent Erdman strain was used with every batch of human macrophages as an internal standard. During the 7-day period, the tubercle bacilli multiplied by a factor of 37. PZA was inhibitory at the lowest concentration tested

Table 1. Growth kinetics of tested Mycobacterium tuberculosis isolates in cultured human macrophages with and without pyrazinamide (PZA) compared with BACTEC minimum inhibitory concentrations (MICs) and pyrazinamidase (PZase) activity.

\begin{tabular}{|c|c|c|c|c|c|c|c|}
\hline \multirow[b]{3}{*}{ Isolate } & \multicolumn{5}{|c|}{ Cultured human macrophages } & \multirow{3}{*}{$\begin{array}{c}\text { BACTEC MIC } \\
\text { of PZA } \\
(\mathrm{mg} / \mathrm{l})\end{array}$} & \multirow[b]{3}{*}{ PZase activity } \\
\hline & \multicolumn{4}{|c|}{ cfu ratio* in presence of $\mathrm{PZA}$ at concentration $(\mathrm{mg} / \mathrm{l})$} & \multirow{2}{*}{$\begin{array}{c}\% \text { inhibition at } \\
\text { day } 7 \text { by } \\
800 \mathrm{mg} / 1 \cdot \mathrm{PZA}\end{array}$} & & \\
\hline & 0 & 50 & 200 & 800 & & & \\
\hline \multicolumn{8}{|l|}{ Laboratory } \\
\hline Erdman & 37 & 3.2 & 2.8 & 1.3 & 96.5 & 50 & Positive \\
\hline $\mathrm{H} 37$ & 35 & 5.2 & 2.5 & 1.2 & 96.6 & 25 & Positive \\
\hline TMC311 & 30 & 21.0 & 31.0 & 33.0 & -10 & $>6400$ & Negative \\
\hline \multicolumn{8}{|l|}{ Clinical } \\
\hline TB186 & 146 & 4.4 & 2.2 & 1.0 & 99.3 & 3200 & Positive \\
\hline TB189 & 87 & 7.4 & 0.7 & 0.1 & 99.9 & $<25$ & Positive \\
\hline TB206 & 16 & 11.9 & 5.1 & 2.5 & 84.4 & 400 & Weakly positive \\
\hline TB213 & 19 & 3.2 & 0.9 & 0.4 & 97.9 & $<25$ & Positive \\
\hline
\end{tabular}

* Colony-forming units (cfu) day $7:$ cfu day 0 . 

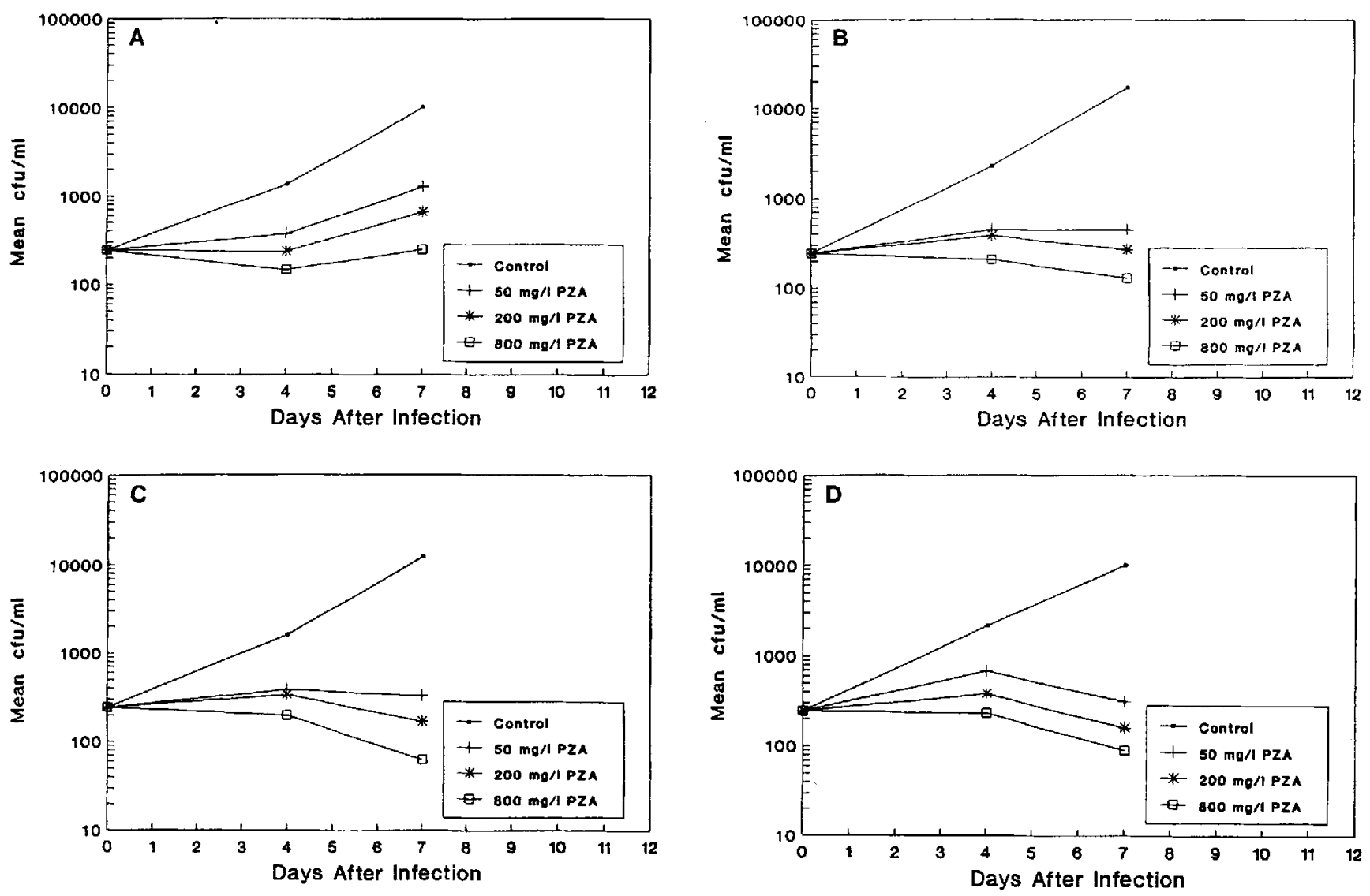

Figure 1. Growth of Erdman strain of Mycobacterium tuberculosis tested with and without pyrazinamide (PZA) in cultured human macrophages under different media: $\mathbf{A}$, traditional macrophage system; $\mathbf{B}$, initial amounts of PZA added daily; $\mathbf{C}$, culture medium with and without PZA replaced daily with fresh medium and constant drug concentrations; $\mathbf{D}$, culture medium with and without PZA replaced daily with conditioned medium.

$(25 \mu \mathrm{g} / \mathrm{ml})$. However, even at $800 \mu \mathrm{g} / \mathrm{ml}$ the drug only slowed intracellular bacillary growth and was not bactericidal. In the absence of PZA the resistant isolate TB206 multiplied 16fold and the susceptible isolate TB189 multiplied 87-fold compared with the initial number of bacilli. At $800 \mu \mathrm{g} / \mathrm{ml}$, the resistant isolate still grew 2.5 -fold, whereas there was a marked bactericidal decrease in numbers of TB189 bacilli. The growth kinetics of all tested $M$. tuberculosis isolates are summarized in table 1 . With increasing PZA concentrations, growth of all except the PZA-resistant TMC311 was diminished.

Ineffectiveness of PZA against different $\mathrm{M}$. bovis $B C G$ strains. During the 10 days of observation, the two BCG strains showed no significant difference between the control and the highest concentration of PZA $(400 \mathrm{mg} / \mathrm{l})$ tested.

Effects of adding PZA to the macrophage media throughout the period of infection. In the four experiments illustrated in figure 1, the Erdman strain grew more between days 4 and 7 than between days 0 and 4 . This suggested that PZA might be losing effectiveness by being metabolized. In the second experiment, the same system was used but additional drug equal to the initial concentration was added daily to the culture medium; for example, 800 became $5600 \mathrm{mg} / 1$ by day 7 (figure 1B). Despite the presence of these large amounts of PZA, no significant decrease in tubercle bacilli was observed during the first 4 days, and only minimal decline by day 7 . Experiments were also done in which the medium was replaced instead of adding PZA daily (figure $1 \mathrm{C}$ ) or in which medium with PZA was taken from cultures of infected macrophages at the end of day 7 of culture and used on freshly infected macrophages (figure ID). In both instances, the PZA was slightly more inhibitory than PZA added just once at the beginning of the experiment or added daily, indicating that its relative loss of effectiveness with time after infection and addition was not due to its breakdown.

Early kinetics of Erdman strain in macrophage cultures. If the change in effectiveness of PZA with time after infection was not due to inactivation of PZA, it might have been due to a change in characteristics of the infecting tubercle bacilli, for example, emergence of resistant clones. This was tested by counts at 8- to 12-h intervals after a single addition of 100 or $400 \mathrm{mg} / 1 \mathrm{PZA}$. After an initial loss of almost $50 \%$ of the 


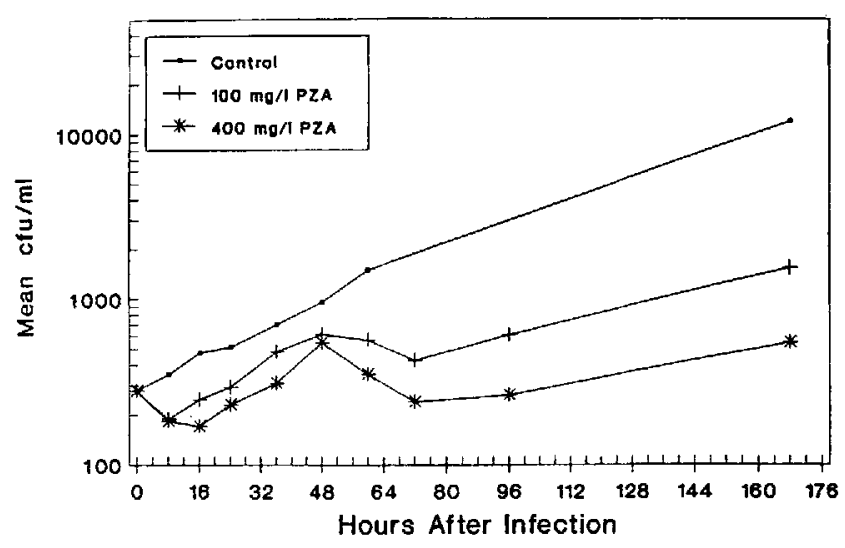

Figure 2. Growth of Erdman strain of Mycobacterium tuberculosis tested with and without pyrazinamide (PZA) in cultured human macrophages. Viable counts were made at 8- to 12-h intervals for 4 days.

viable tubercle bacilli within $16 \mathrm{~h}$, a period of growth independent of PZA concentration followed (figure 2). Within the next $24 \mathrm{~h}$, there was again a decrease in tubercle bacilli in the presence of PZA. At day 3 the bacilli again began to multiply.

Action of POA in macrophage cultures. As shown in figure 3 , POA was not significantly protective at either 50 or 200 $\mathrm{mg} / \mathrm{l}$, compared with $50 \mathrm{mg} / \mathrm{l}$ PZA against the Erdman strain.

PZA MICs in the BACTEC system. The MICs of PZA for seven $M$. tuberculosis isolates varied from $<25$ to $>6400 \mathrm{mg} / \mathrm{l}$ (table 1). One of the BCG strains had an MIC of 6400 and the other, $>6400 \mathrm{mg} / \mathrm{l}$.

PZase activity. All $M$. tuberculosis isolates but one (TMC311) showed PZase activity (table 1). Isolate TB206 was consistently weakly positive. The two $M$. bovis BCG strains were negative.

Effect of PZA against Erdman strain in the BACTEC system. The Erdman strain was tested for comparison of results

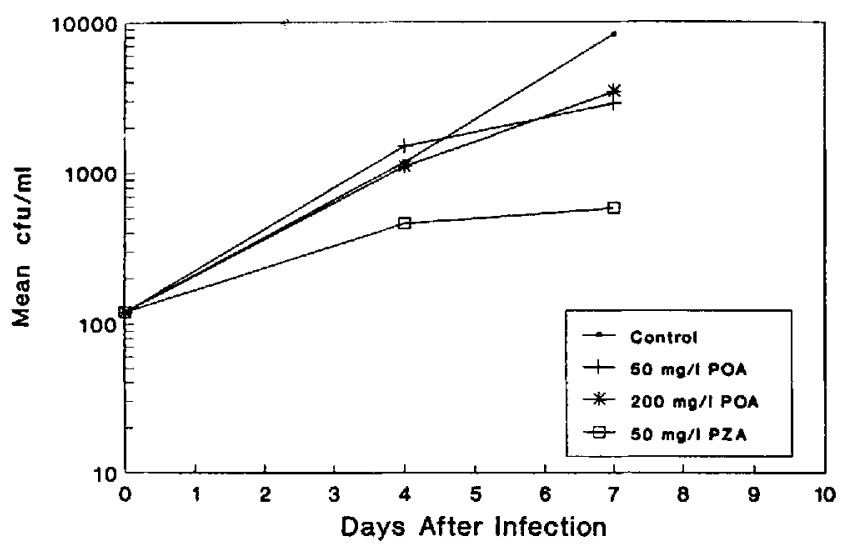

Figure 3. Growth of Erdman strain of Mycobacterium tuberculosis tested with and without pyrazinoic acid (POA) or pyrazinamide (PZA) in cultured human macrophages.
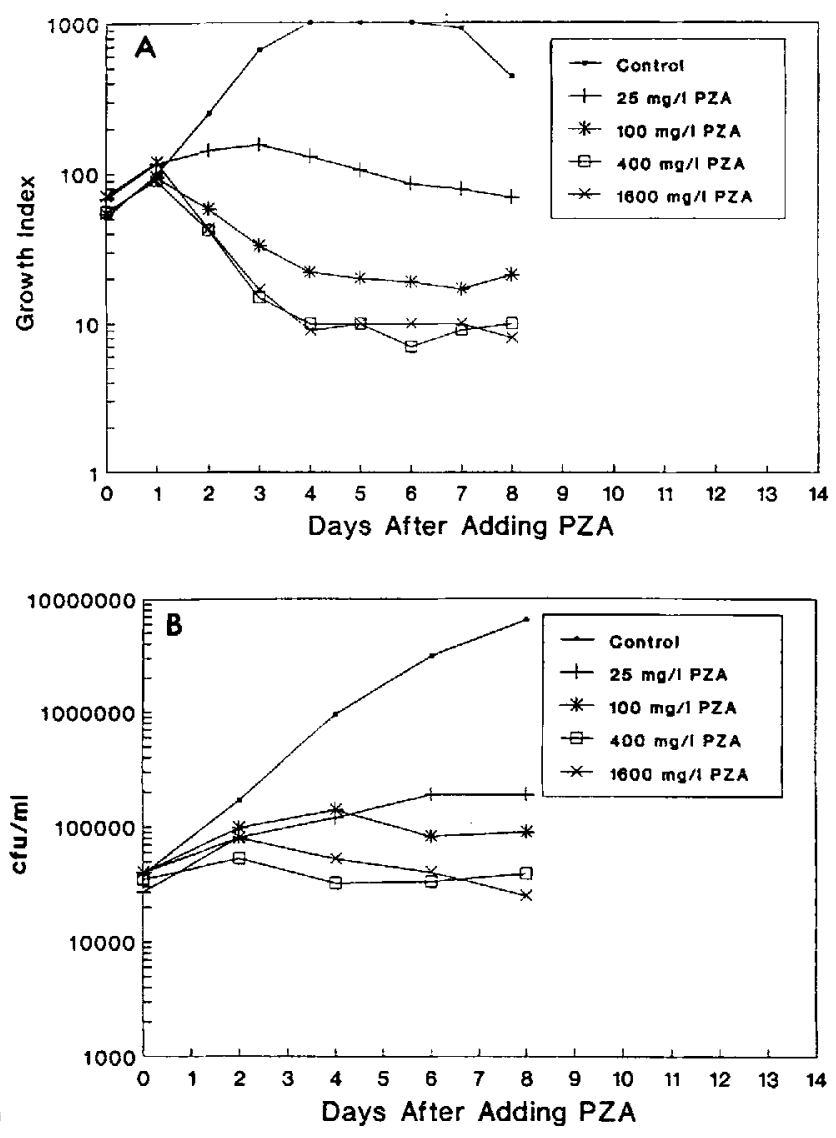

Figure 4. Growth kinetics of Erdman strain of Mycobacterium tuberculosis cultured in special $7 \mathrm{H} 12$ broth with and without pyrazinamide (PZA): A, expressed as growth index in the BACTEC system; $B$, colony-forming units per milliliter.

with those in infected macrophages. Both release of ${ }^{14} \mathrm{CO}_{2}$ and counts were determined. Figure $4 \mathrm{~A}$ shows inhibition of the Erdman strain proportional to PZA concentration up to $400 \mathrm{mg} / \mathrm{l}$. The inhibition was bacteriostatic, as shown by counts of colony-forming units per milliliter (figure 4B).

Action of POA in the BACTEC system against Erdman or TMC311 strain. The ineffectiveness of POA against tubercle bacilli in macrophages might be due to its inability to enter the macrophage. If so, it might be effective if used directly in 7H12 broth. Also, if it is the effector molecule of PZA [9], it should be able to inhibit PZase-negative tubercle bacilli as readily as it does PZase-producing bacilli. These hypotheses were tested with the Erdman strain (PZA-susceptible) and TMC311 (PZA-resistant, PZase-negative). Figure 5 shows that high concentrations of $\mathrm{POA}(\geqslant 400 \mathrm{mg} / \mathrm{l})$ inhibited both strains similarly and in proportion to the concentration of the drug through the highest concentration $(1600 \mathrm{mg} / \mathrm{l})$ used, either at $\mathrm{pH} 6.0$ or 6.8 .

The ability of POA to inhibit tubercle bacilli in $7 \mathrm{H} 12$ broth was greater at $\mathrm{pH} 6.0$ than at $\mathrm{pH} 6.8$. This suggested a connection between $\mathrm{pH}$ and inhibition. Since tubercle bacilli are 

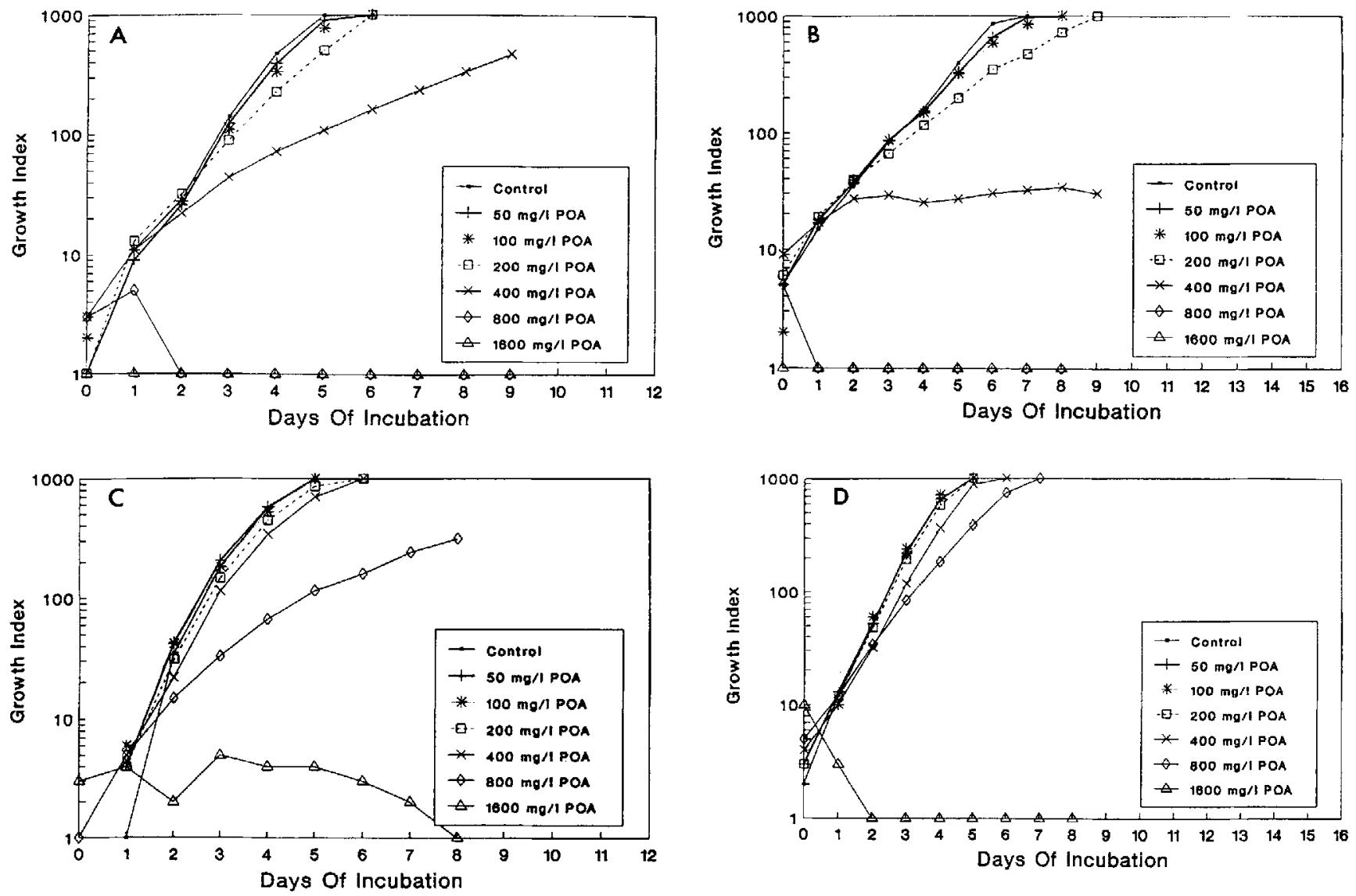

Figure 5. Growth kinetics with and without pyrazinoic acid (POA) of Erdman (pyrazinamide [PZA]-susceptible) strain (A, C) and PZAresistant strain TMC311 (B, D) cultured in special (pH 6.0) and regular (pH 6.8) 7H12 broth, respectively, and expressed as growth index in the BACTEC system.

inhibited by low $\mathrm{pH}$ alone [15], the $\mathrm{pH}$ values of media containing the various concentrations of POA were measured. The larger, inhibitory concentrations of POA caused significant decreases in $\mathrm{pH}$ (figure 6), and these were proportional to the initial $\mathrm{pH}$ of the $7 \mathrm{H} 12$ broth to which the POA was added.

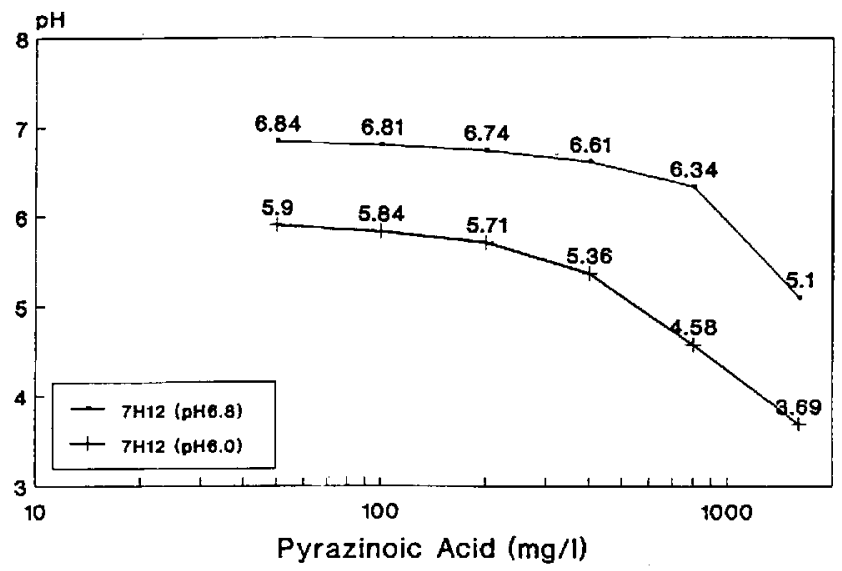

Figure 6. $\mathrm{pH}$ values of regular ( $\mathrm{pH} 6.8$ ) and special ( $\mathrm{pH} 6.0) 7 \mathrm{H} 12$ broth with different amounts of pyrazinoic acid (POA).

\section{Discussion}

A pulmonary tuberculous lesion begins when an inhaled tubercle bacillus is ingested by a resident alveolar macrophage. If this macrophage is incapable of killing the tubercle bacillus, the bacterium replicates, and its progeny eventually lyse the macrophage. Bacilli released from the disintegrated macrophage are then taken up by other alveolar macrophages, which in turn are also lysed. The products of cell and bacterial debris are chemoattractants for circulating monocytes and polymorphs, and in time the monocytes become the primary cell type at the site of the lesion [16].

The mechanisms by which macrophages attempt to kill mycobacteria and the ways by which mycobacteria avoid being killed have been reviewed by Lowrie [16]. The main problem faced in the study of macrophage-killing mechanisms in a host is the complexity of this system.

PZA was synthesized before 1940 [2], but its antituberculous activity was not recognized until the early 1950 s [3]. The inclusion of PZA in treatment regimens has made possible the shortening of therapy to 6 months [6, 7], and at present PZA is the third most important drug in the modern chemotherapy of tuberculosis $[4,5]$. Largely for methodologic reasons, the 
uptake and biotransformation of PZA has been studied only sporadically. There is still a gap in our knowledge of how PZA actually works $[17,18]$.

Because there are individual variations in uptake and killing of tubcrcle bacilli [13] by macrophages, we used the same monocy te donor during the experiments reported here. As an internal standard, the virulent Erdman strain $M$. tuberculosis was used with every batch of macrophages. Our results showed a bacteriostatic effect of PZA on the Erdman strain, confirming recently published data $[19,20]$.

The effectiveness of PZA varied widely among mycobacterial strains. As expected, it was ineffective either in the BACTEC system or in cultured macrophages against PZase-negative $M$. tuberculosis and $M$. bovis BCG. In experiments to determine whether PZA is bactericidal or bacteriostatic, growth of $M$. tuberculosis in $7 \mathrm{H} 12$ broth media provided no evidence for killing by PZA within 1 week of observation. PZA in broth appeared to be bacteriostatic at best (figure 4), whereas POA at high concentrations completely stopped ${ }^{14} \mathrm{CO}_{2}$ production by tubercle bacilli. In cultured macrophages infected with PZA-susceptible strains, however, PZA only slowed bacterial growth unless used in large concentrations not clinically achievable. This confirms our earlier observations [19].

PZA acts synergistically with other antituberculous drugs to kill tubercle bacilli. We have in fact observed this with chloroquine [21]. McDermott and Tompsett [22] recognized that PZA is active only at an acid pH. When PZA is bactericidal in vitro, the $\mathrm{pH}$ it requires is only slightly above the $\mathrm{pH}$ lethal for tubercle bacilli.

Since the growth of the controls varied considerably (table 1), we calculated the percentage of inhibition to enable comparison of PZA activity within the macrophages against the different isolates. Those susceptible were $96.5 \%-99.9 \%$ inhibited, whereas all but one (TB186) of the resistant ones were less so. The anomalous behavior of TB1 86 may be due to metabolic heterogeneity among progeny. On the basis of the percentage of inhibition, there was a good correlation between the MICs determined in vitro and the activity of PZA within human macrophages.

A simple method is available for qualitative determination of PZase activity [14]. With this method, several groups have confirmed a correlation between PZase activity and susceptibility to PZA [23-25]. In this study, we used two isolates with discrepant results: TB206 had weak activity, and TB186 was recognized already as an exception. McClatchy et al. [23] described an isolate that produced PZase but was not proportionally susceptible to PZA. This heterogenous strain showed $50 \%$ resistant colonies on subculture. Butler and Kilburn [26] worked with H37Rv mutants and found no direct correlation between loss of PZase activity and the level of PZA resistance. So far, however, only a few strains have been found that produce PZase but are PZA-resistant.

The metabolism of PZA in humans is well understood [27]. Two of three major metabolites of PZA were identified in 1972 as POA and 5-hydroxy-POA [28]. 5-hydroxy-PZA, the recently recognized third metabolite [29], is produced by the direct action of xanthine oxidase on PZA [30]. Lacroix et al. [31] studied the pharmacokinetics of PZA and its metabolites in healthy people and proposed the following metabolic pathways: major (PZA is deaminated to POA, which is hydroxylated to 5-OH-POA) or minor (PZA is first hydroxylated to 5-OH-PZA and then deaminated to 5-OH-POA). If certain strains of tubercle bacilli could also metabolize PZA by different pathways, it might explain the occasional discrepant results between PZase activity and susceptibility to PZA.

As regards mode of action, the theory that PZA inhibits tubercle bacilli via POA produced by bacterial PZase is consistent with our findings. PZase-negative tubercle bacilli were resistant to PZA in both infected cultured macrophages and the BACTEC system. Although POA was unable to inhibit tubercle bacilli in cultured macrophages, it was able to inhibit them at high concentrations in the BACTEC system. POA's ability to inhibit tubercle bacilli in the BACTEC system was proportional to its concentration and to a decrease in $\mathrm{pH}$ that in itself may be inhibitory (figure 6). It is possible, therefore, that PZA inhibits tubercle bacilli in cultured macrophages physically rather than biochemically. After entering infected macrophages, PZA would be converted to POA by tubercle bacilli and would accumulate in the phagolysosomes, thereby lowering the microenvironmental $\mathrm{pH}$.

The results shown in figure 2 support the hypothesis [19] that the antituberculosis action of PZA is controlled via a selfmodulating feedback loop. In the acidic (pH 5.2) environment of the host's phagolysosomes [32, 33], PZase in tubercle bacilli will convert PZA to POA. If POA cannot reach tubercle bacilli sequestered in the macrophage, as our results suggest, it may be that POA formed intracellularly cannot escape from the macrophage. This would result in accumulation of POA and a drop in $\mathrm{pH}$ sufficient to be toxic for tubercle bacilli. At the same time, however, mycobacterial metabolism produces ammonia, which can increase the intracellular $\mathrm{pH}$ [34], and, to a certain extent, neutralize the acidity of POA. $M$. tuberculosis-derived protein has an effect similar to that of ammonia [35]. Either mechanism would allow resumed growth of the tubercle bacilli within the macrophage.

The comparative activity of PZA and POA against tubercle bacilli in the infected macrophage and broth BACTEC systems provides further insights into the possible mechanism of PZA's clinical effectiveness versus $M$. tuberculosis. Activity of PZA or POA in the BACTEC system is not dependent on drug penetration, as it is in the macrophage model. POA was active in the BACTEC system but not in cultured macrophages, whereas PZA, which can readily enter cultured macrophages, was active in both. Tubercle bacilli were not killed in the BACTEC system, and inhibition by PZA required higher concentrations in the BACTEC system than in cultured macrophages. These results suggest that the cultured macrophage must contribute actively or passively to effectiveness of PZA, 
such as through the proposed mechanism of low $\mathrm{pH}$ generated by PZase in the phagolysosomes. A purely in vitro test such as the BACTEC system can serve as a convenient tool for evaluating antimycobacterial drugs, but the cultured macrophage system may more closely approximate in vivo reality.

\section{References}

1. World Health Organization. Tuberculosis control: report of a joint IUAT/WHO study group. WHO Tech Rep Ser 1982;671:1-26

2. Hall SA, Spoerri PE. Synthesis in the pyrazine series. II. Preparation and properties of amino-pyrazine. J Am Chem Soc 1940;62:664-665

3. Yeager RL, Munroe WGC, Dessau FI. Pyrazinamide (aldinamide) in the treatment of pulmonary tuberculosis. Am Rev Tuberc 1952; 65: 523-546

4. Perez-Stable EJ, Hopewell PC. Chemotherapy of tuberculosis. Semin Respir Med 1988;9:459-469

5. Leff DR, Leff AR. Tuberculosis control policies in major metropolitan health departments in the United States. IV. Standards in 1988. Am Rev Respir Dis 1989;139:1350-1355

6. East and Central African/British Medical Research Council Fifth Collaborative Study. Controlled clinical trial of 4 short-course regimens of chemotherapy (three 6-month and one 8-month) for pulmonary tuberculosis: final report. Tubercle 1986;67:5-15

7. Hong Kong Chest Service/British Medical Research Council. Five-year follow-up of a controlled trial of five 6-month regimens of chemotherapy for pulmonary tuberculosis. Am Rev Respir Dis 1987;136: 1339-1342

8. Mitchison DA. Treatment of tuberculosis. J R Coll Physicians Lond 1980;14:91-99

9. Konno K, Feldmann FM, McDermott W. Pyrazinamide susceptibility and amidase activity of tubercle bacilli. Am Rev Respir Dis 1967; 95:461-469

10. Salfinger M, Reller LB, Demchuk B, Johnson ZT. Rapid radiometric method for pyrazinamide susceptibility testing of Mycobacterium tuberculosis. Res Microbiol 1989;140:301-309

11. Crowle AJ. The tubercle bacillus-human macrophage relationship studied in vitro. In: Friedman H, Bendinelli M, eds. Mycobacterium tuberculosis: interactions with the immune system. New York: Plenum Publishing, 1988:99-135

12. Crowle AJ, Elkins N, May MH. Effectiveness of ofloxacin against Mycobacterium tuberculosis and Mycobacterium avium, and rifampin against $M$. tuberculosis in cultured human macrophages. Am Rev Respir Dis 1988;137:1141-1146

13. Crowle AJ, May MH. Replication of lyophilized and cultured BCG in human macrophages. Am Rev Respir Dis 1983;128:673-679

14. Wayne LG. Simple pyrazinamidase and urease tests for routine identification of mycobacteria. Am Rev Respir Dis 1974;109:147-151

15. Mitchison DA. The action of antituberculosis drugs in short-course chemotherapy. Tubercle 1985;66:219-225

16. Lowrie DB. Mononuclear phagocyte-mycobacterium interaction. In: Ratledge $\mathrm{C}$, Stanford J, eds. The biology of the mycobacteria. Vol 2. New York: Academic Press, 1983:235-278

17. Zierski M. Pharmakologie, Toxikologie und klinische Anwendung von Pyrazinamid. Prax Klin Pneumol 1981;35:1075-1105
18. Hawkins JE. Drug susceptibility testing. In: Kubica GP, Wayne LG, eds. The mycobacteria: a sourcebook. Part A. New York: Marcel Dekker, 1984:177-193

19. Crowle AJ, Sbarbaro JA, May MH. Inhibition by pyrazinamide of tubercle bacilli within cultured human macrophages. Am Rev Respir Dis 1986;134:1052-1055

20. Crowle AJ, Salfinger M, May MH. 1,25(OH $)_{2}$-vitamin $D_{3}$ synergizes with pyrazinamide to kill tubercle bacilli in cultured human macrophages. Am Rev Respir Dis 1989;139:549-552

21. Crowle AJ, May MH. Chloroquine, a potentially new antituberculosis drug [abstract 888]. In: Program and abstracts of the 29th Interscience Conference on Antimicrobial Agents and Chemotherapy (Houston). Washington, DC: American Society for Microbiology, 1989

22. McDermott W, Tompsett $\mathbf{R}$. Activation of pyrazinamide and nicotinamide in acidic environments in vitro. Am Rev Tuberc 1954;70:748-754

23. McClatchy JK, Tsang AY, Cernich MS. Use of pyrazinamidase activity in Mycobacterium tuberculosis as a rapid method for determination of pyrazinamide susceptibility. Antimicrob Agents Chemother 1981; 20:556-557

24. Schröder KH, der Arbeitskreis Mykobakterien. Die Pyrazinamidase von M. tuberculosis. Möglichkeit der Empfindlichkeitsprüfung gegen Pyrazinamid. Prax Klin Pneumol 1985;39:170-172

25. Salfinger M, Kafader FM. Susceptibility testing of Mycobacterium tuberculosis to pyrazinamide. Zentralbl Bakteriol Mikrobiol Hyg [A] 1987;265:404-407

26. Butler WR, Kilburn JO. Susceptibility of Mycobacterium tuberculosis to pyrazinamide and its relationship to pyrazinamidase activity. Antimicrob Agents Chemother 1983;24:600-601

27. Yü TF, Berger L, Stone DJ, Wolf J, Gutman AB. Effect of pyrazinamide and pyrazinoic acid on urate clearance and other discrete renal functions. Proc Soc Exp Biol Med 1957;96:264-267

28. Weiner IM, Tinker JP. Pharmacology of pyrazinamide: metabolic and renal function studies related to the mechanism of drug-induced urate retention. J Pharmacol Exp Ther 1972;180:411-434

29. Yamamoto T, Moriwaki Y, Takahashi S, Hada T, Higashino K. 5hydroxypyrazinamide, a human metabolite of pyrazinamide. Biochem Pharmacol 1987;36:2415-2416

30. Yamamoto T, Moriwaki Y, Takahashi S, Hada T, Higashino K. Rapid and simultaneous determination of pyrazinamide and its major metabolites in human plasma by high-performance liquid chromatography. J Chromatogr 1987;413:342-346

31. Lacroix C, Hoang TP, Nouveau J, Guyonnaud C, Laine G, Duwoos H, Lafont $\mathrm{O}$. Pharmacokinetics of pyrazinamide and its metabolites in healthy subjects. Eur J Clin Pharmacol 1989;36:394-400

32. Sprick MG. Phagocytosis of $M$. tuberculosis and $M$. smegmatis stained with indicator dyes. Am Rev Tuberc 1956;74:552-565

33. Jacques $\mathrm{YV}$, Bainton DF. Changes in $\mathrm{pH}$ within the phagocytic vacuoles of human neutrophils and monocytes. Lab Invest 1978;39:179-185

34. Ohkuma S, Poole B, Fluorescence probe measurement of the intralysosomal $\mathrm{pH}$ in living cells and the perturbation of $\mathrm{pH}$ by various agents. Proc Natl Acad Sci USA 1978;75:3327-3331

35. Chicurel M, García E, Goodsaid F. Modulation of macrophage lysosomal $\mathrm{pH}$ by Mycobacterium tuberculosis-derived proteins. Infect Immun 1988;56:479-483 